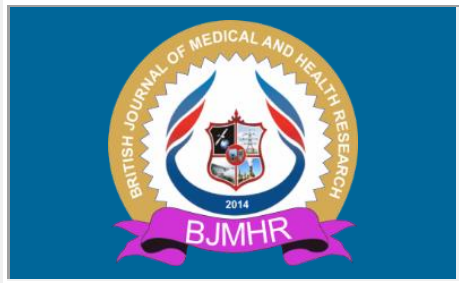

\title{
BJMHR
}

British Journal of Medical and Health Research

Journal home page: www.bjmhr.com

\section{An Exploratory Hospital-Based Study On Somatic Status and Dietary Habits of Patients with Pancreatitis}

\author{
Akshatha Nagaraja Thantry ${ }^{1}$, Asna Urooj*1 \\ 1.Department of Food Science and Nutrition, University of Mysore, Manasagangothri, \\ Mysore, Karnataka, 570006
}

\section{ABSTRACT}

Nutritional status of pancreatitis patients varies among new cases and recurrent patients. Under nutrition is one of the major risk factors in the mortality and morbidity of patients with pancreatitis. Severe malnutrition will adversely affect outcomes, as occurs in other critical diseases. Malnutrition is known to occur in $50-80 \%$ of chronic alcoholics and alcohol is a major etiological factor. Excess body fat, lack of lean body mass, muscle wasting and poor immune status are some of the indicators of malnutrition associated with poor prognosis in patients. This study was an observational prospective study conducted on 22 subjects in two multispecialty hospitals of Mysore city in the period of 2 months. Patients admitted to the hospital with diagnosis of pancreatitis and who were above 17 years of age were recruited for the study. Questionnaire was designed and data such as demographic profile, anthropometric, biochemical, clinical symptoms, medications, $24 \mathrm{hr}$ dietary recall of hospital and home were collected. Results were expressed as Mean \pm Standard deviation. Statistical analysis was conducted using student $t$ test and chi square test by means of SPPS 16.0 software. Among 22 subjects, 17 were diagnosed with acute pancreatitis and 5 with chronic pancreatitis, 19 subjects had a history of chronic alcoholism. The serum protein levels was in the lower end of normal $(6.73 \pm 0.93 \mathrm{~g} / \mathrm{dl})$ while liver enzymes and pancreatic enzymes were high(AST-75 $\pm 81.28 \mathrm{U} / \mathrm{L}$, ALT-47.11 \pm 48.44 U/L, P.lipase-485.18 \pm 431.65 , P.amylase-667.45 \pm 824.53 ). The mean weight on admission was $62.2 \pm 9.59 \mathrm{~kg}$, a weight loss of 2-4 kg was seen among majority of the patients at discharge, which could be attributed to the low-calorie intake (mean $367 \pm 488 \mathrm{kcal}$ ) during hospital stay. Even though the mean BMI was $23.41 \pm 4.17$, the weight was attributed to empty calories acquired by daily alcohol consumption rather than diet. The mean intake of home recall was $1507 \pm 288$ and that of hospital recall was $367 \pm 488$. Malnutrition was found to be present among subjects and significant difference (p value-0.000) was seen in patients' calorie intake. Nutritional status plays a major role in prognosis of patients and early nutrition support must be practiced for better recovery, reduction in length of stay and improvement in nutritional status of the patient.

Keywords: Pancreatitis, Nutritional status, dietary recall, Malnutrition, Acute Pancreatitis, Chronic Pancreatitis

*Corresponding Author Email: asnaurooj@ foodsci.uni-mysore.ac.in

Received 05 November 2020, Accepted 15 December 2020

Please cite this article as: Thantry A et al., An Exploratory Hospital-Based Study On Somatic Status and Dietary Habits of Patients with Pancreatitis. British Journal of Medical and Health Research 2020. 


\section{INTRODUCTION}

Nutritional status of pancreatitis patients varies among new cases and recurrent pancreatitis patients. Under nutrition is one of the major risk factors in the mortality and morbidity of patients with pancreatitis. Malnutrition occurs in 50-80\% of chronic alcoholics and alcohol is a major etiological factor. Acute pancreatitis (AP) is a common severe illness of the digestive tract with variable involvement of other regional tissues and/or remote organ system. ${ }^{1}$ Mild disease is associated with minimal organ dysfunction and rapid recovery, while severe disease is associated with multiple organ system failure and local complications such as necrosis, abscess, fistulas and pseudocystformation. ${ }^{2}$ Chronic pancreatitis (CP) is an inflammatory condition of the pancreas that lead to endocrine and exocrine insufficiency. ${ }^{3}$ Nutritional support in the patients with AP is both very critical and more complex. The treatment of AP is essentially supportive as there is no available modality to reverse the progression once initiated. It is increasingly being recognized that maintenance of gut integrity is the most important determinant of outcome in AP. ${ }^{4}$ It is one of the most common diseases of the gastrointestinal tract, leading to tremendous emotional, physical, and financial human burden. Recent evidences show the incidence of AP varies between 4.9 and 73.4 cases per 100,000 worldwide. An increase in the annual incidence for AP has been observed in most recent studies. ${ }^{5}$ The two most common etiological factors, representing more than $80 \%$ of cases, are gallstones and alcohol abuse. Overall, about $15 \%$ to $20 \%$ of patients, AP progresses to a severe illness with an extended disease course. These severely ill patients may develop organ failure and/or local complications such as pancreatic necrosis. ${ }^{6}$ Prospective and population-based studies on the incidence of pancreatitis are scarce in India. ${ }^{7}$ Very limited data is available on the nutritional management of pancreatitis in Indian scenario. Enteral and Total Parenteral nutrition are reported to be used in the management of severe pancreatitis during hospitalization. ${ }^{8}$

During acute pancreatitis, specific and non-specific metabolic changes occur. Under the influence of inflammatory mediators and pain, the basal metabolic rate may increase leading to a higher energy demand. If pancreatitis is complicated by sepsis, roughly 80 percent of the patients are in a hypermetabolic state with an increase in the resting energy expenditure (REE). These patients have increased nutrient requirements due to increased rates of REE and protein breakdown. A negative nitrogen balance has been associated with an adverse clinical outcome. Net nitrogen losses are as much as 20-40 g/day in some patients with acute pancreatitis. Deficiencies in certain amino acids may increase pancreatic inflammation, leading to a potential vicious circle. ${ }^{9}$ Micronutrient and vitamin deficiencies (such as hypocalcemia, hypomagnesemia, lower zinc levels, and thiamine and folate deficiencies), may also be present on admission or develop during hospitalization. ${ }^{9,10}$ Anorexia secondary to abdominal pain, 
Nausea and vomiting, alcohol and other substance abuse and diabetes also contribute to malnutrition in patients. ${ }^{11}$

\section{Rationale of this study:}

To understand the overall nutritional status of patients with Pancreatitis and to identify major etiology for Pancreatitis and their dietary behavior in Mysore city.

\section{MATERIALS AND METHOD}

\section{Study Design:}

This study was an observational prospective study conducted on 22 subjects in two multispecialty hospitals of Mysore city in a period of 2 months. Informed consent was taken and study abided by the ethical guidelines given by the Institutional Ethics committee [IHEC-UOM No.159/Ph.D/2018-19.

Data was collected using standardized questionnaire that included demographic profile, modified Kuppuswamy score for socio economic status, anthropometric data including height, weight, BMI and recent weight loss and loss of appetite were noted. Habits such as alcohol consumption and nicotine abuse were noted. Biochemical parameters pertaining to Pancreas functioning and inflammatory markers like ALP and Serum Protein, Albumin and Globulin were collected from medical record. One day dietary recall of home and hospital was recorded using $24 \mathrm{hr}$ dietary recall.

\section{Selection criteria:}

1. Patients diagnosed with either Acute or Chronic Pancreatitis

2. Age group of $17-74 \mathrm{yrs}$

Results were expressed as mean \pm standard deviation. Statistical analysis was conducted using SPSS 16.0 software and analysis like student $t$ test and chi square test were carried out. P value less than 0.05 were accepted as statistically significant.

\section{RESULTS AND DISCUSSION}

Table 1: Demographic data of Subjects

\begin{tabular}{lllll}
\hline Study parameters & \multicolumn{2}{l}{ Subjects(n) } & Total(n) \\
\hline Education & Primary & Higher Primary & PUC & \\
& 15 & 2 & 5 & 22 \\
Socio Economic Status & Low & Middle & Upper & \\
& 17 & 3 & 2 & 22 \\
Area of Residence & Urban & Rural & & \\
& 9 & 13 & & 22 \\
Type of Diet & Mixed & Veg & & 22 \\
& 21 & 1 & None & \\
Personal habits & Alcohol & Smoking, alcohol & 3 & 22 \\
\hline
\end{tabular}


Table 1 depicts the demographic data of the subjects. Majority of subjects were uneducated and had less than primary education $(n=15)$. Most of the patients were from low socioeconomic status and were from rural areas $(n=17)$. Majority of the subjects consumed mixed type of diet $(n=21)$. Out of 22 subjects- 14 subjects had the habit of both smoking and alcohol consumption and 5 were reported to only consume alcohol. Only 3 subjects did not have any habits. Most of the patients were observed to consume low cost local brands of alcohol available in the small road side bar. The diagnosis and related clinical parameters are given in Table number 2 .

Table 2: Diagnosis and diagnosis related parameter

\begin{tabular}{llll}
\hline Study Parameter & Subjects(n) & Total(n) \\
\hline Diagnosis & AP* & CP' & \\
& 17 & 05 & 22 \\
Mean Age(min-max) yrs & $40.7(17-74)$ & $39.7(25-48)$ & \\
Weight loss & & & \\
$<2 \mathrm{~kg}$ & 14 & 2 & 22 \\
2-4kg & 3 & 3 & \\
Mean wt loss & $1.4 \mathrm{~kg}$ & & \\
\hline
\end{tabular}

*AP- Acute Pancreatitis, ${ }^{+}$CP- Chronic Pancreatitis

Etiology can be majorly divided into acute pancreatitis (17) and chronic pancreatitis (5). Major etiology was alcohol induced acute pancreatitis (n-10), followed by pseudocyst of pancreas (n6), chronic Pancreatitis (n-5) and gall stone induced pancreatitis (n-1). There was no significance between age and type of pancreatitis, while age varied between $17 \mathrm{yr}$ to $74 \mathrm{yr}$. Average weight loss of patients were noted to be $1.4 \mathrm{~kg}$ in the past one month. Significant association between age, weight and BMI, were observed ( $\mathrm{p}$ value-0.000). As the age increased, adiposity and thus weight and BMI significantly increased as seen in table 3.

Table 3: Correlation between Age, weight and BMI

*BMI- Body Mass Index, ${ }^{\text {WT- }}$ weight

\begin{tabular}{lll}
\hline \multicolumn{2}{l}{ Variable } & P VALUE \\
\hline Age & BMI & $0.019^{*}$ \\
& WT $^{+}$ & $0.043^{*}$ \\
BMI* $^{*}$ & WT & $0.000^{* * *}$ \\
& AGE & $0.019^{*}$ \\
\hline
\end{tabular}

Table 4 Symptoms with relation to type of Diagnosis

\begin{tabular}{|c|c|c|c|c|c|}
\hline & \multicolumn{4}{|c|}{ Subjects(n) } & Total(n) \\
\hline \multirow[t]{2}{*}{ Appetite } & \multicolumn{4}{|c|}{ Normal poor } & \multirow{3}{*}{22} \\
\hline & 3 & & 19 & & \\
\hline Vomiting & No & Yes & Yes- $B^{\ddagger}$ & Yes- $\mathrm{H}^{\S}$ & \\
\hline $\mathrm{AP} *$ & 9 & 5 & 2 & 1 & 22 \\
\hline $\mathrm{CP}^{+}$ & & 1 & 0 & 0 & \multirow{5}{*}{22} \\
\hline Diarrhea & \multicolumn{2}{|c|}{ Yes } & No & & \\
\hline $\mathrm{AP}$ & \multicolumn{2}{|c|}{2} & 14 & & \\
\hline $\mathrm{CP}$ & \multicolumn{2}{|l|}{1} & 4 & & \\
\hline Dyspnea & \multicolumn{2}{|c|}{ Yes } & No & & \\
\hline
\end{tabular}




\begin{tabular}{llll}
\hline $\mathrm{AP}$ & 3 & 14 & 22 \\
$\mathrm{CP}$ & 0 & 5 & \\
\hline
\end{tabular}

*AP- Acute Pancreatitis, +CP- Chronic Pancreatitis, ${ }^{\ddagger}$ Yes-B- Bilious Vomiting, ${ }^{\S}$ Yes-HHemetemesis.

Table no.4 depicts symptoms with relation to type of diagnosis. Symptoms of diseases like vomiting - bilious $(B)(n=6)$, non-bilious $(\mathrm{NB})$ or haemtemesis $(H)$, presence or absence of diarrhea $(n=3)$, presence or absence of dyspnea $(n=3)$ were observed, AP patients had more symptoms than CP. Most of the patients complained of poor appetite $(n=19)$ due to pain and nausea. Pain score ranges from 0 - no pain to 10 worst pain possible, this scale is Wong-Baker's Facial Grimace Scale. All the patients suffered from pain (AP-6.7 \pm 1.39 and CP-7.4 \pm 0.89 ) and was the primary reason for admission. Biochemical parameters of subjects are presented in table no.5. Hb levels were observed to vary among types of pancreatitis and ranged from 7.6 to $18.4 \mathrm{gm} / \mathrm{dl}$ (mean-13.36 \pm 2.94 ). Both liver enzymes were increased in both type of pancreatitis and AST was higher in both the cases (mean-75.83 \pm 85.90 ). The levels of protein were substantially low and the mean value $(6.69 \pm 0.99)$ was closer to lower normal end because of malnutrition and catabolism. Random blood glucose values were collected, the mean values $(122.49 \pm 42.21)$ were within normal range, although only 2 patients had the diagnosis of Diabetes Mellitus. Pancreatic lipase and amylase were observed to be elevated $(485.18 \pm 431.65$ and $667.45 \pm 824.53$ respectively) in both the groups at admission. Comparatively even if it was not significant, patients with chronic pancreatitis had lesser values when compared to acute pancreatitis. Mean nutrient intake during home and hospital stay is tabulated in table no.6. The energy intake at hospital were found to be comparatively less, as the patient is allowed to consume only clear liquids and or soups until the pain subsides (mean-367 \pm 488 ). The energy intakes of home recall were near normal $(1507 \pm 288)$ even if the intake of protein and other nutrients were reduced. Protein intake were found to be low even at home recall (38 \pm 19$)$ as the patients belonged to low socio-economic status and were consuming less diversified food and high biological value protein food, while hospital diet lacked protein due to liquid diet given for long time(mean- $8 \pm 14$ ). Fat intake were found to be substantially low in both the recalls (home-27 \pm 8 , hospital-6.4 \pm 8.5 ) due to intake of liquid diet. Significant differences in nutrient intake were observed between home recall and hospital recall ( $p$ value-0.000).

Table 5 Biochemical Parameters of subjects

\begin{tabular}{lllll}
\hline Blood Parameters & $\begin{array}{l}\text { Normal } \\
\text { Range }\end{array}$ & Mean \pm SD & Mean \pm SD $(\mathbf{n}=22)$ \\
\cline { 3 - 4 } & M-13.8-17.2 & $13.7 \pm 2.84$ & $12.04 \pm 3.04$ & $13.36 \pm 2.94$ \\
\hline $\mathrm{Hb}^{*}(\mathrm{gm} / \mathrm{dL})$ & $\mathrm{F}-12.1-15.1$ & & $\mathbf{C P}(\mathbf{5})$ & \\
& $6-8$ & $6.73 \pm 0.93$ & $6.58 \pm 1.31$ & $6.69 \pm 0.99$ \\
Serum Protein $(\mathrm{gm} / \mathrm{dL})$ & $80-140$ & $124.78 \pm 43.97$ & $114.74 \pm 39.03$ & $122.49 \pm 42.21$ \\
$\mathrm{RBS}^{\dagger}(\mathrm{mg} / \mathrm{dL})$ & & & & \\
Enzymes $_{\text {P.Lipase }}^{\ddagger}(\mathrm{U} / \mathrm{L})$ & $0-64$ & $554.36 \pm 453.52$ & $250.00 \pm 256.91$ & $485.18 \pm 431.65$ \\
\hline
\end{tabular}




\begin{tabular}{lllll} 
P. Amylase $^{\S}(\mathrm{U} / \mathrm{L})$ & $0-86$ & $787.77 \pm 906.6$ & $258.69 \pm 105.2$ & $667.45 \pm 824.53$ \\
AST $^{\|}(\mathrm{U} / \mathrm{L})$ & $7-55$ & $75 \pm 81.28$ & $78.10 \pm 110.92$ & $75.83 \pm 85.90$ \\
ALT $^{\mathbb{I}}(\mathrm{U} / \mathrm{L})$ & $8-48$ & $47.11 \pm 48.44$ & $28.13 \pm 13.27$ & $42.80 \pm 43.44$ \\
ALP(IU/L) & $44-147$ & & & $122.60 \pm 62.91$ \\
CRP(mg/L) & $<10$ & & & $39.30 \pm 50.53$ \\
\hline
\end{tabular}

${ }^{*}$ Hb-Haemoglobin, ${ }^{+}$RBS- Random Blood Glucose, ${ }^{\ddagger} \mathrm{P}$. Lipase- Pancreatic Lipase, ${ }^{\S} \mathrm{P}$. Amylase- Pancreatic Amylase,;AST-Aspartate Transaminase; "IILT-Alanine amino Transferase.

Table 6: Mean Nutrient Intake at Hospital and Home.

\begin{tabular}{llll}
\hline Nutrients & $\begin{array}{l}\text { Home Diet } \\
(\text { Mean } \pm \text { SD) }\end{array}$ & $\begin{array}{l}\text { Hospital diet } \\
(\text { Mean } \pm \text { SD) }\end{array}$ & P value \\
\hline Protein $(\mathrm{g})$ & $38 \pm 19$ & $8 \pm 14$ & $0.000^{* * *}$ \\
Fat $(\mathrm{g})$ & $27 \pm 8$ & $6.4 \pm 8.5$ & $0.000^{* * *}$ \\
Carbohydrate(g) & $270 \pm 61$ & $63 \pm 80$ & $0.000^{* * *}$ \\
Energy(kcal) & $1507 \pm 288$ & $367 \pm 488$ & $0.000 * * *$ \\
\hline
\end{tabular}

\section{DISCUSSION:}

In this study it was observed that, most of the patients were malnourished. Malnutrition played a major role in prognosis of patients. Weight loss was observed during the course of hospital stay as well as during the course of the disease. Screening of malnutrition helps in early intervention and thus better prognosis during the course of disease. ${ }^{12}$ Demographic data showed that most of the patients were from rural background and were with or without minimum education. The subjects belonged to low socio-economic status and thus most of them used local brand of alcohol for daily consumption. Although type of alcohol might not be studied so far, but this might be the major reason for early development of pancreatitis in younger age groups. Only three subjects were reported to have no habit of alcohol or smoking. Thus, major etiology for pancreatitis was alcohol consumption and majority were admitted with acute pancreatitis when compared to chronic pancreatitis.

This study observed that symptoms were prominently seen in acute pancreatitis than chronic pancreatitis. Majority of the symptoms were seen like vomiting, diarrhea, dyspnea, loss of appetite while patients with $\mathrm{CP}$ had lesser symptoms and better tolerance towards pain. Although serum protein values were normal, it was in the lower level of normal range. Liver enzymes and pancreatic enzymes were elevated while blood sugars were in normal range for most of the patients. The inflammatory markers like ALP, C-RP were out of range and had a varied result.

Nutrient intake was very low during the course of the hospital stay and home recall, thus malnutrition was prominent. Even though calorie intake was near normal range, this was due to daily consumption of alcohol, attributing to empty calories. Protein intake was noticed to be less than half of the RDA. During the hospital stay, the intake of nutrients was restricted due 
to nil per oral status of the patients due to symptoms and were fed only with clear liquids for next 4-5 days until symptoms subsided. Patients were allowed to take soft diet only after 7-8 days of admission and this led to further weight loss and malnutrition.

Thus, our study revealed major etiological factor that is alcohol consumption, we also observed the main reason for malnutrition in hospital stay being under feeding due symptoms and pain. However, the study had few limitations (1) the study was conducted for short duration and with small number, (2) Longitudinal study would help in understanding the progression in disease with respect to dietary intake, (3) use of specific nutrition screening tool along with functional assessment is necessary to assess and monitor nutritional status.

\section{CONCLUSION:}

Nutrition plays a vital role in prognosis of patients with pancreatitis. Even with smaller group of patients, there was significance seen with age, BMI and weight. Patients were from low socio-economic background and were exposed to local brands of alcohol at an early age of 20 yr. This was the major etiology seen in patients with pancreatitis. Acute pancreatitis patients had more complications like increased pancreatic enzymes, ALP, symptoms like pain, diarrhea, dyspnea and vomiting. Weight loss was observed in almost all the patients and were also experienced loss of appetite due to pain and nausea. Serum protein although in lower range of normal was observed, patients were malnourished due to alcohol consumption and low socioeconomic status leading to further deterioration and poor prognosis. Two patients succumbed to complications like sepsis and MODS during hospital stay. Thus, nutritional intervention at the earliest is of great importance. Patients were fed with only kitchen-based soups and clear liquids which are deficient in major nutrients required. Even though the intake of nutrients at home were likely to be satisfactory, empty calories were derived from daily intake of alcohol in majority of the patients (n-19). Thus, malnutrition has already set in majority of the patients even before admission to hospital.

\section{REFERENCES:}

1. Bradley, E. L. (1993). A clinically based classification system for acute pancreatitis: summary of the International Symposium on Acute Pancreatitis, Atlanta, Ga, September 11 through 13, 1992. Arch Surg, 128(5), 586-590.

2. Shu, Z. J., Li, W. Q., Wang, X. B., Wang, Z. M., Wang, S. H., Wang, L., ... \& Li, J. S. (1998). Gastrointestinal tract complications in severe acute pancreatitis. World J Gastroentero, 4(Suppl 2), 98-99.

3. Mandalia, A., Wamsteker, E. J., \&Di Magno, M. (2018). Recent advances in understanding and managing acute pancreatitis. F1000Research, 7. 
4. Srinivasan G, Venkatakrishnan L, Sambandam S, Singh G, Kaur M, Janarthan K, John B J. Current concepts in the management of acute pancreatitis. J Family Med Prim Care 2016;5:752-8

5. Tenner, S., Baillie, J., DeWitt, J., \& Vege, S. S. (2013). American College of Gastroenterology guideline: management of acute pancreatitis. The American journal of gastroenterology, 108(9), 1400.

6. Spanier, B. M., Bruno, M. J., \& Mathus-Vliegen, E. M. (2011). Enteral nutrition and acute pancreatitis: a review. Gastroenterology research and practice, 2011.

7. Baig, S. J., Rahed, A., \&Sen, S. (2008). A prospective study of the aetiology, severity and outcome of acute pancreatitis in Eastern India. Trop Gastroenterol, 29(1), 20.

8. Negi, N., Mokta, J., Sharma, B., Sharma, R., Jhobta, A., Bodh, V., \&Ranjan, A. (2018). Clinical Profile and Outcome of Acute Pancreatitis: A Hospital-Based Prospective Observational Study in Subhimalayan State. Journal of The Association of Physicians of India, 66, 22.

9. Meier, R., Ockenga, J., Pertkiewicz, M., Pap, A., Milinic, N., Macfie, J., ...\&Keim, V. (2006). ESPEN guidelines on enteral nutrition: pancreas. Clinical Nutrition, 25(2), 275284.

10. Rebours, V. (2015). Role of Enteral and Parenteral Nutrition. Pancreapedia: The Exocrine Pancreas Knowledge Base.

11. Lohr, J. M., Dominguez-Munoz, E., Rosendahl, J., Besselink, M., Mayerle, J., Lerch, M. M.,\& Keller, J. (2017). United European Gastroenterology evidence-based guidelines for the diagnosis and therapy of chronic pancreatitis (HaPanEU). United European Gastroenterology Journal, 5(2), 153-199.

12. Márta, K., Farkas, N., Szabó, I., Illés, A., Vincze, Á.,Pár, G., \& Mosztbacher, D. (2016). Meta-analysis of early nutrition: the benefits of enteral feeding compared to a nil per oral diet not only in severe, but also in mild and moderate acute pancreatitis. International Journal Of Molecular Sciences, 17(10), 1691.

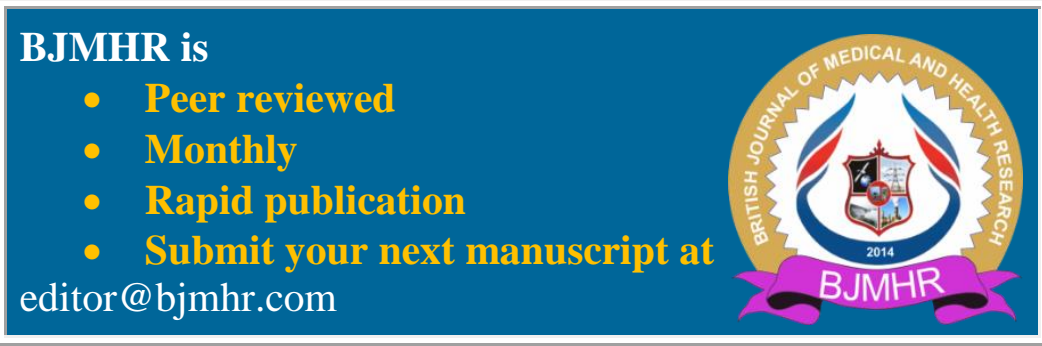

